\title{
A TYCHONOFF ALMOST REALCOMPACTIFICATION
}

\section{R. GRANT WOODS ${ }^{1}$}

\begin{abstract}
Let $X$ be a Tychonoff topological space. A Tychonoff almost realcompact space $a X$ is constructed that contains $X$ as a dense subspace and has the property that if $f: X \rightarrow Y$ is continuous and $Y$ is Tychonoff and almost realcompact, then $f$ can be extended continuously to $a X$. Several characterizations of $a X$ are given, and the relationships between $a X$, the Hewitt realcompactification $v X$, and the minimal $c$-realcompactification $u X$ are investigated. Properties of the projective covers of these spaces, and their relation to $v E(X)(E(X)$ denotes the projective cover of $X)$, are discussed.
\end{abstract}

1. Introduction. In [3], Frolik calls a Hausdorff topological space $X$ almost realcompact if, given any ultrafilter $\mathscr{U}$ of open subsets of $X$ such that $\bigcap_{n \in N} \operatorname{cl}_{X} U_{n} \neq \varnothing$ for each countable subfamily $\left(U_{n}\right)_{n \in N}$ of $\mathscr{U}$, then $\bigcap\left\{\mathrm{cl}_{X} U: U \in \mathscr{U}\right\} \neq \varnothing$. Frolik proved that the topological product of an arbitrary family of almost realcompact spaces is almost realcompact, and that a closed subspace of a regular almost realcompact space is almost realcompact. It follows from these results (and a theorem of Herrlich and van der Slot [7]) that corresponding to each Tychonoff space $X$ there exists an almost realcompact space $a X$ with the following properties: $X \subseteq a X \subseteq \beta X$ (the Stone-Čech compactification of $X$ ), and if $f$ is a continuous function from $X$ into any Tychonoff almost realcompact space $Y$, then $f$ can be continuously extended to a function $f^{a}: a X \rightarrow Y$. In $\$ 2$ we obtain a characterization of $a X$ and discuss some of its properties. In $\S 3$ we discuss the relationship between $a X$ and the $c$-realcompactification $u X$ of $X$ (see [2]), and consider the properties of the projective covers of these spaces. Finally, a comparison is made between $a X$ and the Liu-Strecker almost realcompactification $\rho X$ that lies between $X$ and its Katětov $H$-closed extension (see [8]). The notation and terminology of [4] are used throughout.

Received by the editors March 19, 1973.

AMS (MOS) subject classifications (1970). Primary 54D99; Secondary 54D60, 54G05.

Key words and phrases. Almost realcompactification, Tychonoff space, projective cover, $c$-realcompactification.

${ }^{1}$ This research was partially supported by a grant from the National Research Council of Canada. 
A subset $A$ of a topological space $X$ is regular closed if $A=\mathrm{cl}_{X}\left(\right.$ int $\left._{X} A\right)$. The family $\mathscr{R}(X)$ of all regular closed subsets of $X$ is a complete Boolean algebra under the following operations:

(1) $A \leqq B$ iff $A \subseteq B$,

(2) $\bigvee_{\alpha} A_{\alpha}=\mathrm{cl}_{X}\left[\bigcup_{\alpha} A_{\alpha}\right]$,

(3) $\wedge_{\alpha} A_{\alpha}=\mathrm{cl}_{X} \operatorname{int}_{X}\left[\bigcap_{\alpha} A_{\alpha}\right]$,

(4) $A^{\prime}=\mathrm{cl}_{X}(X-A)$.

It is immediate that Tychonoff almost realcompact spaces can be characterized as follows:

1.1 THEOREM. A Tychonoff space $X$ is almost realcompact if and only if each ultrafilter on $\mathscr{R}(X)$ with the countable intersection property (C.I.P.) has nonempty intersection.

To conclude our introductory remarks, we briefly describe the projective cover, or absolute, of a Tychonoff space. A more detailed discussion may be found in [11]. Recall that a Hausdorff space is extremally disconnected if each of its open subsets has an open closure. If $X$ is a Tychonoff space, then the Stone space $E(\beta X)$ of the Boolean algebra $\mathscr{R}(\beta X)$ is a compact extremally disconnected Hausdorff space whose points are ultrafilters on $\mathscr{R}(\beta X)$. If $A \in \mathscr{R}(\beta X)$, let $\lambda(A)=\{\alpha \in E(\beta X): A \in \alpha\}$; then the map $A \rightarrow \lambda(A)$ is a Boolean algebra isomorphism from $\mathscr{R}(\beta X)$ onto the Boolean algebra of open-and-closed subsets of $E(\beta X)$. Define $k: E(\beta X) \rightarrow \beta X$ as follows: if $\alpha \in E(\beta X)$, put $k(\alpha)=\bigcap\{A: A \in \alpha\}$. Then (see [5]) $k$ is a welldefined continuous function from $E(\beta X)$ onto $\beta X, k$ is irreducible (i.e. proper closed subsets of $E(\beta X)$ are mapped onto proper closed subsets of $\beta X$ by $k$ ), and $k[\lambda(A)]=A$ for each $A \in \mathscr{R}(\beta X)$. Now $k^{\leftarrow}[X]$ is a dense, extremally disconnected, $C^{*}$-embedded subspace of $E(\beta X)$, and the restriction $k_{X}$ of $k$ to $k^{\leftarrow}[X]$ is a perfect irreducible map from $k^{\leftarrow}[X]$ onto $X$. The space $k^{\leftarrow}[X]$ can thus be identified with the projective cover $E(X)$ of $X$ (in the category of Tychonoff spaces and perfect maps) discussed by Strauss in [10]. Evidently $E(\beta X)=\beta E(X)$, and if $X \subseteq T \subseteq \beta X$, then $k^{\leftarrow}[T]=E(T)$.

2. The construction of $a X$. The following result of Herrlich and van der Slot appears as a corollary of Theorem 1 of [7].

2.1 THEOREM. Let $\mathscr{P}$ be a topological property (of Tychonoff spaces) with the following properties:

(a) If each member of a family $\mathscr{F}$ of topological spaces has $\mathscr{P}$, then the product space $\prod\{F: F \in \mathscr{F}\}$ has $\mathscr{P}$.

(b) If $X$ has $\mathscr{P}$ and $S$ is a closed subspace of $X$, then $S$ has $\mathscr{P}$.

(c) Compact spaces have $\mathscr{P}$. 
Then for each Tychonoff space X there exists a "maximal $\mathscr{P}$-extension" of $X$, denoted by $\gamma X$, with the following properties:

(1) $\gamma X$ is a Tychonoff space containing a dense copy of $X$.

(2) $\gamma X$ has $\mathscr{P}$.

(3) If $Y$ is a Tychonoff space with $\mathscr{P}$ and if $f: X \rightarrow Y$ is continuous, then $f$ can be continuously extended to a function $f^{\gamma}: \gamma X \rightarrow Y$.

(4) If $\bar{\gamma} X$ is another space satisfying (1)-(3), there is a homeomorphism from $\gamma X$ onto $\bar{\gamma} X$ that fixes $X$ pointwise.

(5) $\gamma X$ can be identified with the intersection of all subspaces of $\beta X$ that contain $X$ and have $\mathscr{P}$.

As an immediate consequence of the above theorem, we derive the following result.

2.2 THEOREM. Corresponding to each Tychonoff space $X$ there exists a Tychonoff almost realcompact space $a X$ with the following properties:

(1) $X \subseteq a X \subseteq \beta X$.

(2) If $f$ is a continuous function from $X$ to a Tychonoff almost realcompact space $Y$, then $f$ has a continuous extension $f^{a}$ that maps aX into $Y$.

In fact $a X$ is the intersection of all the almost realcompact subspaces of $\beta X$ that contain $X$, and $f^{a}$ is the restriction to aX of the Stone extension $f^{\beta}$ of $f$ that maps $\beta X$ into $\beta Y$.

ProOF Let $\mathscr{P}$ be the topological property "almost realcompact and Tychonoff". Theorem 7 of [3] says that condition (a) of 2.1 is fulfilled for this $\mathscr{P}$, while Theorem 5 of [3] says that condition (b) of 2.1 is fulfilled. As each compact space obviously is almost realcompact, claims (1) and (2) above immediately follow from 2.1. Since $f^{\beta} \mid a X$ and $f^{a}$ both map $a X$ into $\beta Y$ and agree on $X$, they are equal.

Theorem 2.2 tells us that $a X$ exists and that $X \subseteq a X \subseteq \beta X$; it does not tell us which points of $\beta X-X$ will be found in $a X$. As an analogy, the Hewitt realcompactification $v X$ of $X$ consists of those points $p$ of $\beta X$ such that the $z$-ultrafilter on $X$ that converges to $p$ has C.I.P. We wish to derive a similar characterization of $a X$. We begin with some technical lemmas. The first is a well-known result that follows easily from 8.7 of [4].

2.3 Theorem. Let $X$ be a Tychonoff space. Then $v X=\{p \in \beta X$ : each $G_{\delta}$-set of $\beta X$ that contains $p$ meets $\left.X\right\}$.

Let $X$ be a Tychonoff space. An ultrafilter $\mathscr{A}$ on $\mathscr{R}(X)$ is said to converge to a point $p \in \beta X$ if $\{p\}=\bigcap\left\{\mathrm{cl}_{\beta X} A: A \in \mathscr{A}\right\}$. Evidently $\mathscr{A}$ converges to $p$ if and only if $k(\alpha)=p$, where $k: E(\beta X) \rightarrow \beta X$ is the map defined in $\S 1$ and $\alpha=\left\{\mathrm{cl}_{\beta X} A: A \in \mathscr{A}\right\}$. Let $a_{1} X$ denote the set $\{p \in \beta X$ : there exists an ultrafilter $\mathscr{A}$ on $\mathscr{R}(X)$ with C.I.P. that converges to $p\}$. If $n$ is a positive 
integer greater than 1 , we define $a_{n} X$ inductively as follows: $a_{n} X=$ $a_{1}\left(a_{n-1} X\right)$.

We shall need the following result, which appears as 2.18 of [12].

2.4 Lemma. Let $X$ be a Tychonoff space. Then

$$
v E(X)=\{\alpha \in E(\beta X):\{A \cap X: A \in \alpha\} \text { has C.I.P. }\} \text {. }
$$

2.5 Lemma. Let $X$ be a Tychonoff space. Let $k: E(\beta X) \rightarrow \beta X$ be the canonical map defined in $\S 1$. Then $k[v E(X)]=a_{1} X$.

Proof. Let $\alpha \in v E(X)$. Then $\{A \cap X: A \in \alpha\}$ has C.I.P. by 2.4, and converges to $k(\alpha)$. Hence $k(\alpha) \in a_{1} X$. Conversely, if $p \in a_{1} X$, find an ultrafilter $\mathscr{A}$ on $\mathscr{R}(X)$ such that $\mathscr{A}$ has C.I.P. and $\mathscr{A}$ converges to $p$. Put $\alpha=\left\{\mathrm{cl}_{\beta X} A: A \in \mathscr{A}\right\}$. Then $\mathscr{A}=\{A \cap X: A \in \alpha\}$, so $\alpha \in v E(X)$ by 2.4 . Evidently $k(\alpha)=p$, so $p \in k[v E(X)]$. The lemma follows.

We shall need the following result which appears, among other places, as Theorem 1.7 of [1].

2.6 THEOREM. The Tychonoff space $X$ is almost realcompact if and only if $E(X)$ is realcompact.

We need one more technical lemma, which perhaps is of independent interest.

2.7 Lemma. Let $X$ be a Tychonoff space, and let $\left(T_{n}\right)_{n \in N}$ be a countable family of realcompact spaces such that $X \subseteq T_{n} \subseteq \beta X$ for each $n \in N$. Then $\bigcup_{n \in N} T_{n}$ is realcompact.

Proof. Put $Y=\bigcup_{n \in N} T_{n}$. As $X \subseteq Y \subseteq \beta X$, it follows that $\beta Y=\beta X$ (see 6.7 of [4]). Hence to show that $Y$ is realcompact, it suffices to show that if $p \in \beta X-Y$, then there is a $G_{\delta}$-set of $\beta X$ containing $p$ and disjoint from $Y$. But if $p \in \beta X-Y$, then $p \in \beta X-T_{n}$ for each $n \in N$, so as $\beta T_{n}=$ $\beta X$ (since $X \subseteq T_{n} \subseteq \beta X$ ), there exists a $G_{\delta}$-set $G_{n}$ of $\beta X$ such that $p \in G_{n}$ and $G_{n} \cap T_{n}=\varnothing$. Put $G=\bigcap_{n \in N} G_{n}$. Then $G$ is a $G_{\delta}$-set of $\beta X$ containing $p$ and disjoint from $Y$. Hence $Y$ is realcompact.

2.8 THEOREM. Let $X$ be a Tychonoff space. Then $a X=\bigcup_{n \in N} a_{n} X$.

Proof. Repeated use of Lemma 2.5 shows that $k\left[v E\left(a_{n} X\right)\right]=a_{n+1} X$ and $v E\left(a_{n} X\right) \subseteq E\left(a_{n+1} X\right) \subseteq v E\left(a_{n+1} X\right)$ for each $n \in N$. Thus

$$
E\left(\bigcup_{n \in N} a_{n} X\right)=k^{\leftarrow}\left[\bigcup_{n \in N} a_{n} X\right]=\bigcup_{n \in N} E\left(a_{n} X\right)=\bigcup_{n \in N} v E\left(a_{n} X\right) .
$$

By Lemma 2.7, $\bigcup_{n \in N} v E\left(a_{n} X\right)$ is realcompact. Hence by 2.6, $\bigcup_{n \in N} a_{n} X$ is almost realcompact. Hence $a X \subseteq \bigcup_{n \in N} a_{n} X$. 
Each realcompact space is almost realcompact (see Theorem 10 of [3]), and each almost realcompact extremally disconnected space is realcompact (see Theorem 1.2 of [1]). Hence $a E(X)=v E(X)$. But the extension of the mapping $k_{X}: E(X) \rightarrow X$ to $a E(X)$ maps $a E(X)$ into $a X$ (see 2.2); hence by $2.5 a_{1} X \subseteq a X$. It then follows from 2.2 that $a\left(a_{1} X\right)=a X$. A repetition of this argument shows that $a_{n} X \subseteq a X$ for each $n \in N$. Hence $\bigcup_{n \in N} a_{n} X \subseteq a X$, and so $\bigcup_{n \in N} a_{n} X=a X$.

It is natural to conjecture that $a X=a_{1} X$ for any Tychonoff space $X$. I have been unable either to prove this conjecture or find a counterexample to it. More generally, it is possible that for each Tychonoff space $X$, $a X=a_{n} X$ for some positive integer $n$ (perhaps depending on $X$ ). It is evident that if $a_{n} X$ is almost realcompact for some $n \in N$, then $a_{k} X=a_{n} X$ for each $k \geqq n$.

We now consider some of the properties of $a X$. Note that since each realcompact space is almost realcompact, $a X \subseteq v X$ for each Tychonoff space $X$.

2.9 THEOREM. Let $X$ be a Tychonoff space. Then:

(i) $v X$ is not locally compact at any part of $v X-a_{1} X$.

(ii) $\beta X-v X$ is dense in $\beta X-a X$.

(iii) If $v X$ is locally compact then $a_{1} X=a X=v X$.

Proof. (1) In Theorem 2.8 of [12] it is shown that $E(\beta X)-E(v X)$ is dense in $E(\beta X)-v E(X)$. Applying the map $k$, and noting that $\beta X-a_{1} X \subseteq$ $k[E(\beta X)-v E(X)]$, we conclude that $\beta X-v X$ is dense in $\beta X-a_{1} X$. Thus $\operatorname{cl}_{\beta X}(\beta X-v X)=\mathrm{cl}_{\beta X}\left(\beta X-a_{1} X\right)$. But $\beta X-\mathrm{cl}_{\beta X}(\beta X-v X)=\{p \in v X: v X$ is locally compact at $p\}$ (see 1.10 of [11]). Hence (i) follows. Statement (ii) now follows from the fact that $\beta X-v X \subseteq \beta X-a X \subseteq \beta X-a_{1} X$, and statement (iii) follows immediately from statement (i).

Some attention has been devoted to finding conditions on a pair of Tychonoff spaces $X$ and $Y$ that are equivalent to the truth of the equation $v(X \times Y)=v X \times v Y$. A general solution of this problem has not been obtained. The following result relates this question to the corresponding question about almost realcompactifications.

2.10 THeorem. Let $X$ and $Y$ be Tychonoff spaces. If $v(X \times Y)=$ $v X \times v Y$, then $a(X \times Y)=a X \times a Y$.

Proof. In general $X \times Y \subseteq a(X \times Y) \subseteq v(X \times Y)$. Since $v X \times v Y=$ $v(X \times Y) \subseteq \beta(X \times Y)$, it follows that $a X \times a Y$ is an almost realcompact subspace of $\beta(X \times Y)$ that contains $X \times Y$. Hence $a(X \times Y) \subseteq a X \times a Y$, so both $X \times a Y$ and $a(X \times Y)$ are contained in $a X \times a Y$. If $(p, q) \in X \times a Y-$ $a(X \times Y)$, then since $\{p\} \times a Y$ and $a(X \times Y)$ are almost realcompact subspaces of the regular almost realcompact space $a X \times a Y$, it follows that 
their intersection is almost realcompact (see Theorem 7 of [3]; note that regularity is needed). Thus $[\{p\} \times a Y] \cap[a(X \times Y)]$ is almost realcompact and is properly contained in $\{p\} \times a Y$ while containing $\{p\} \times Y$. This is a contradiction, so it follows that $X \times a Y \subseteq a(X \times Y)$; evidently $a(X \times a Y)=$ $a(X \times Y)$. A repetition of this argument (with $a X \times a Y$ in place of $X \times a Y$ ) yields that $a X \times a Y \subseteq a(X \times Y)$. Hence $a X \times a Y=a(X \times Y)$.

3. Almost realcompactifications and $c$-realcompactifications. A Tychonoff space $X$ is said to be $c$-realcompact if for each point $p \in \beta X-X$ there exists a normal lower semicontinuous function $f$ on $\beta X$ (see [9]) such that $f(p)=0$ and $f$ is positive on $X$. Dykes defined $c$-realcompact spaces in [2]; they are discussed in some detail in [6]. The following result may be found in 1.1 and 2.5 of [6].

3.1 THEOREM. Let $X$ be a Tychonoff space. Let $u X=\{p \in \beta X$ : each ultrafilter on $\mathscr{R}(X)$ that converges to $p$ has C.I.P.\}. Then:

(1) $u X$ is the smallest c-realcompact space between $X$ and $\beta X$.

(2) $X$ is c-realcompact if and only if given $p \in \beta X-X$, there exists a decreasing sequence $\left(A_{n}\right)_{n \in \mathrm{N}} \subseteq \mathscr{R}(\beta X)$ such that $p \in \bigcap_{n \in \mathrm{V}} A_{n}$ and $\bigcap_{n \in N} A_{n} \cap X=\varnothing$.

The space $u X$ is called the $c$-realcompactification of $X$.

It is proved in 3.3 of [2] that each almost realcompact space is $c$-realcompact. Hence if $X$ is a Tychonoff space, then $X \subseteq u X \subseteq a X \subseteq v X$. The relationship between $u X$ and $a X$ is clarified in the following.

3.2 Lemma. Let $X$ be a Tychonoff space. Then:

(a) $a X$ is the smallest space $T$ between $X$ and $\beta X$ such that $E(T)$ is realcompact. In particular, $v E(X) \subseteq E(a X)$.

(b) $u X$ is the largest space $T$ between $X$ and $\beta X$ such that $E(T) \subseteq v E(X)$.

Proof. Recall that $X \subseteq T \subseteq \beta X$ iff $E(X) \subseteq E(T) \subseteq E(\beta X)=\beta E(X)$. Part (a) now follows from 2.2 and 2.6.

Let $\alpha \in E(u X)$. Then $\alpha$ is an ultrafilter on $\mathscr{R}(\beta X)$ such that $\{A \cap X$ : $A \in \alpha\}$ converges to $k(\alpha) \in u X$. Thus $\{A \cap X: A \in \alpha\}$ has C.I.P., and so $\alpha \in v E(X)$ by 2.4 . Hence $E(u X) \subseteq v E(X)$. Conversely, if $p \in \beta X-u X$, there is an ultrafilter $\alpha$ on $\mathscr{R}(\beta X)$ such that $\{A \cap X: A \in \alpha\}$ converges to $p$ but does not have C.I.P. Hence $\alpha \notin v E(X)$ so $k^{\leftarrow}(p)-v E(X) \neq \varnothing$. Hence (b) holds.

3.3 THEOREM. The following conditions on a Tychonoff space $X$ are equivalent:

(a) $v E(X)=E(T)$ for some $T$ such that $X \subseteq T \subseteq \beta X$.

(b) $v E(X)=E(a X)$.

(c) $u X=a X$.

(d) $u X=a_{1} X$. 
Proof. $\quad(\mathrm{a}) \Rightarrow(b)$ : If $v E(X)=E(T)$ for some $T$ such that $X \subseteq T \subseteq \beta X$, then $E(T)$ is realcompact so $T$ is almost realcompact. Hence $a X \subseteq T$. But by 3.2(a), $v E(X) \subseteq E(a X)$, so $T \subseteq a X$. Hence $T=a X$.

(b) $\Rightarrow$ (c): Evidently $E(a X) \subseteq v E(X)$ so, by $3.2(\mathrm{~b}), a X \subseteq u X$. Hence $a X=u X$.

(c) $\Rightarrow$ (d): In general $u X \subseteq a_{1} X \subseteq a X$, so if $u X=a X$ then $u X=a_{1} X$.

(d) $\Rightarrow$ (a): By $2.5, k[v E(X)]=a_{1} X$ so $v E(X) \subseteq E\left(a_{1} X\right)$. Thus $E(u X) \subseteq$ $v E(X) \subseteq E\left(a_{1} X\right)=E(u X)$ so $v E(X)=E(u X)$.

An example of a Tychonoff space $X$ such that $u X \neq a X$ can be found in the example on pp. 240-241 of [9]. It is constructed as follows: let $T$ be the Tychonoff plank (see 8.20 of [4]), let $A=W^{*} \times\{\omega\}$ and $B=$ $\left\{\omega_{1}\right\} \times N^{*}$ denote, respectively, the top and right edge of $T^{*}$. Let $X^{*}$ denote the space obtained from $T^{*} \times N$ by identifying $A \times\{2 n-1\}$ with $A \times\{2 n\}$ and identifying $B \times\{2 n\}$ with $B \times\{2 n+1\}$. Let $t$ denote the corner point $\left(\omega_{1}, \omega, n\right)$ of $X^{*}$, and put $X=X^{*}-\{t\}$. Mack and Johnson show in [9] that $X^{*}=v X$ and that $X$ is not weak $c b$ (see $\S 3$ of [9]) while $X^{*}$ is weak $c b$. In Theorem 1.11 of [6] it is shown that $X$ is weak $c b$ iff $u X$ is weak $c b$. Hence $X=u X$, i.e. $X$ is $c$-realcompact.

Let $f: T^{*} \times N \rightarrow X^{*}$ denote the identification map described above. Then $f$ takes $T \times N$ onto $X$. Obviously $T^{*} \times N=v(T \times N)$, so by 2.9(iii), $a(T \times N)=T^{*} \times N$. Hence the extension of $f \mid T \times N$ to $T^{*} \times N$ maps $T^{*} \times N$ into $a X$. But $f\left[T^{*} \times N\right]=X^{*}=v X$, so $a X=v X \neq u X$.

We conclude this paper by comparing the Strecker-Liu almost realcompactification $\rho X$ of $X$ (see [8]) to $a X$ and $u X$. Recall that the Katětov $H$-closed extension $\kappa X$ of the Hausdorff space $X$ is formed as follows: Let $Y$ be the family of all open ultrafilters $\mathscr{U}$ on $X$ such that

$$
\bigcap\left\{\mathrm{cl}_{X} U: U \in \mathscr{U}\right\}=\varnothing,
$$

and let $\kappa X=X \cup Y$, topologized as follows: open subsets of $X$ remain open in $\kappa X$, and if $p \in Y$ then $\{\{p\} \cup G: G \in p\}$ is a neighbourhood base at $p$ in $\kappa X$. Then $\kappa X$ is a Hausdorff space that is a closed subspace of each Hausdorff space in which it can be embedded (i.e. $\kappa X$ is $H$-closed), and if $h X$ is any other $H$-closed Hausdorff space that contains $X$ as a dense subspace, then there is a continuous map from $\kappa X$ onto $h X$ that fixes $X$ pointwise. Let $\rho X=X \cup\left\{p \in Y: \bigcap_{n \in N} \mathrm{cl}_{X} G_{n} \neq \varnothing\right.$ for each countable subfamily $\left(G_{n}\right)_{n \in N}$ of $\left.p\right\}$. Liu and Strecker prove that $\rho X$ (endowed with the subspace topology inherited from $\kappa X$ ) is an almost realcompact Hausdorff space with the property that if $S$ is an an almost realcompact Hausdorff space that contains $X$ as a dense subspace, then $\rho X$ can be mapped into $S$ by a continuous function that fixes $X$ pointwise. Thus $\rho X$ plays the same role in the class of Hausdorff spaces as $a X$ plays in the class of Tychonoff spaces. 
The relation between $\rho X, a_{1} X$, and $a X$ is described in the following theorem.

3.4 THEOREM. Let $X$ be a Tychonoff space and let $f: \kappa X \rightarrow \beta X$ be the unique continuous function that is the identity on $X$. Then:

(1) $f[\rho X]=a_{1} X$.

(2) $\rho X=f^{\leftarrow} f[\rho X]$ if and only if $u X=a_{1} X$.

Proof. (1) It is easily seen that, if $p \in \kappa X-X$, then

$$
f(p)=\bigcap\left\{\mathrm{cl}_{\beta X} U: U \in p\right\} .
$$

If $p \in \rho X-X$, then $\left\{\operatorname{cl}_{X} U: U \in \mathscr{U}\right\}$ is an ultrafilter on $\mathscr{R}(X)$ with C.I.P. that converges to $f(p) \in \beta X$. Thus $f(p) \in a_{1} X$. Conversely, if $q \in a_{1} X$, then find an ultrafilter $\mathscr{A}$ on $\mathscr{R}(X)$ with C.I.P. such that $\mathscr{A}$ converges to $q$. Let $p=\left\{V: V\right.$ is open in $X$ and int ${ }_{X} A \subseteq V$ for some $\left.A \in \mathscr{A}\right\}$. Then $p \in \rho X$ and $f(p)=q$. Hence $f[\rho X]=a_{1} X$.

(2) Assume that $\rho X=f^{\leftarrow}[f[\rho X]]=f^{\leftarrow}\left[a_{1} X\right]$. Let $q \in a_{1} X$ and let $\mathscr{A}$ be any ultrafilter on $\mathscr{R}(X)$ that converges to $q$. Let $p=\{V: V$ is open in $X$ and int $_{X} A \subseteq V$ for some $\left.A \in \mathscr{A}\right\}$. Then $p \in \kappa X$ and $f(p)=q$. Thus $p \in$ $f^{\leftarrow}\left[a_{1} X\right]=\rho X$. Hence $\mathscr{A}$ has C.I.P., and so each ultrafilter on $\mathscr{R}(X)$ that converges to $q$ has C.I.P. Hence $q \in u X$ and so $a_{1} X \subseteq u X$. But $u X \subseteq a_{1} X$ in general, so $u X=a_{1} X$. Conversely, if $u X=a_{1} X$, let $p \in f^{\leftarrow}\left[a_{1} X\right]=f^{\leftarrow}[u X]$. Then the ultrafilter $\left\{\mathrm{cl}_{X} V: V \in p\right\}$ on $\mathscr{R}(X)$ converges to $f(p) \in u X$. Thus this ultrafilter has C.I.P., and so $p \in \rho X$. Thus $f^{\leftarrow} f[\rho X]=\rho X$.

\section{REFERENCES}

1. N. Dykes, Mappings and realcompact spaces, Pacific J. Math. 31 (1969), 347-358. MR 41 \#7644.

2. - Generalizations of realcompact spaces, Pacific J. Math. 33 (1970), 571 581. MR 43 \#2668.

3. Z. Frolik, A generalization of realcompact spaces, Czechoslovak Math. J. 13 (88) (1963), 127-138. MR 27 \#5224.

4. L. Gillman and M. Jerison, Rings of continuous functions, University Series in Higher Math., Van Nostrand, Princeton, N.J., 1960. MR 22 \#6994.

5. A. M. Gleason, Projective topological spaces, Illinois J. Math. 2 (1958), 482-489. MR 22 \#12509.

6. K. Hardy and R. G. Woods, On c-realcompact spaces and locally bounded normal functions, Pacific J. Math. 43 (1972), 647-656.

7. H. Herrlich and J. van der Slot, Properties which are closely related to compactness, Nederl. Akad. Wetensch. Proc. Ser A 70=Indag. Math. 29 (1967), 524-529. MR 36 \#5898.

8. C. T. Liu and G. E. Strecker, Concerning almost realcompactifications, Czechoslovak Math. J. 22 (1972), 181-190.

9. J. E. Mack and D. G. Johnson, The Dedekind completion of $C(X)$, Pacific J. Math. 20 (1967), 231-243. MR 35 \#2150. 
10. D. P. Strauss, Extremally disconnected spaces, Proc. Amer. Math. Soc. 18 (1967), 305-309.

11. R. G. Woods, Co-absolutes of remainders of Stone-Cech compactifications, Pacific J. Math. 37 (1971), 545-560.

12. _ Ideals of pseudocompact regular closed sets and absolutes of Hewitt realcompactificalions, General Topology and Appl. 2 (1972), 315-331.

Department of Mathematics, University of Manitoba, Winnipeg, Canada 